\title{
Estudio de un caso de enseñanza de materias STEM a través del ecourbanismo apoyado por herramientas avanzadas de diseño, en el horizonte 2030 de objetivos de desarrollo sostenible (ODS)
}

\section{Study of a case of teaching STEM subjects through ecourbanism supported by advanced design tools, in the 2030 horizon of sustainable development goals (SDGs).}

\author{
Francisco del Cerro Velázquez. \\ Universidad de Murcia. Murcia, España \\ fcerro@um.es \\ Fernando Lozano Rivas. \\ Universidad de Murcia. Murcia, España \\ fernando.lozano@um.es
}

\section{Resumen}

En el artículo se propone una práctica innovadora en la enseñanza-aprendizaje de las materias STEM, en concreto se propone el ecourbanismo como instrumento metodológico apoyado en herramientas de diseño avanzadas (GIMP, Goolzoom y Sketchup) y, a su vez, se justifica su contribución, desde la educación, a la penetración de los Objetivos de Desarrollo Sostenible en la sociedad. En este sentido, la resolución 70/01 de la Asamblea General de las Naciones Unidas propone la agenda 2030 para el Desarrollo Sostenible. Se formulan 17 Objetivos de Desarrollo Sostenible (ODS) necesarios para alcanzar dicho estado de equilibrio. En el artículo se justifica la importancia del ODS 4 (Educación de Calidad) para alcanzar, expandir e implantar de forma efectiva el resto de ODS y se evidencia la potencialidad del ecourbanismo como instrumento metodológico que desde la educación permitirá esta idea. Para ello se analiza cómo, con el ecourbanismo y su relación intrinseca con la educación ambiental, es posible trabajar en el aula los ODS y alcanzar los objetivos de las materias curriculares favoreciendo el desarrollo de las nuevas generaciones, con una actitud crítica y positiva con el Desarrollo Sontenible. De acuerdo con lo expuesto, se relacionan los objetivos de desarrollo sostenible con los objetivos ecourbanísticos de una ecociudad. Finalmente, se aplica esta práctica innovadora mediante el siguiente caso: Proyección y desarrollo de espacios de oportunidad en un centro educativo; proyecto mediante el cual, alumnos de la asignatura de Dibujo Técnico $1 .^{\circ}$ de bachillerato del colegio La Inmaculada de Cartagena, participaron en la I Olimpiada de Arquitectura de la region de Murcia (2018) "Make Cool Your School” (2018).

Palabras clave

STEM; Ecourbanismo; Objetivos de Desarrollo Sostenible; Educación de Calidad; Educación Ambiental; ODS 4.

\begin{abstract}
The article proposes an innovative practice in the teaching-learning of STEM subjects, specifically proposes ecourbanism as a methodological tool supported by advanced design tools (GIMP, Goolzoom and Sketchup) and, in turn, justifies its contribution, from education, to the penetration of the Sustainable Development Goals in society. In this sense, resolution 70/01 of the United Nations General Assembly proposes the Agenda 2030 for Sustainable Development. It formulates 17 sustainable development goals (SDGs) necessary to achieve this state of equilibrium. The article justifies the importance of SDG 4 (Quality Education) to achieve, expand and effectively implement the rest of the SDGs and demonstrates the potential of ecourbanism as a methodological instrument that from education will allow this idea. To this end, it is analyzed
\end{abstract}


how, with ecourbanism and its intrinsic relationship with environmental education, it is possible to work the SDGs in the classroom and achieve the objectives of the curricular subjects, favoring the development of new generations, with a critical and positive attitude towards Sustainable Development. In accordance with the above, sustainable development goals are related to the ecourban goals of an ecocity. Finally, this innovative practice is applied through the following case: Projection and development of opportunity spaces in a school; project through which, $1 .^{\circ}$ high school's students of technical drawing of the school La Inmaculada de Cartagena, participated in the First Olympiad of Architecture of the region of Murcia (2018) "Make Cool Your School" (2018).

\section{Keywords}

STEM; Ecourbanism; Sustainable Development Goals; Quality Education; Environmental Education; SDG 4.

\section{Introducción}

Anticipamos que existen numerosos antecedentes de urbanistas que afirman que los modelos de urbanismo estándar o conocidos como tradicionales no consiguen dar soluciones a los problemas sociales, económicos y ambientales de las ciudades (Chueca Goitia, 2011) y, por ello, se hace necesario introducir nuevos modelos (ecourbanos) basados en distintos parámetros, capaces de dar respuesta a los problemas antes citados. Sin embargo, no se ha dado el salto que se plantea en la innovación propuesta, trasladar el ecourbanismo a la educación y, aunque se consultaron urbanistas para la reciente redacción de los Objetivos de Desarrollo Sostenible, tampoco se han relacionado estos objetivos con los parámetros del ecourbanismo.

El desarrollo sostenible es, sin lugar a dudas, un concepto del que se ha escrito, comentado e investigado a lo largo del tiempo.

El 25 de septiembre de 2015 se lleva a cabo en la ONU un proyecto ambicioso y transformativo en el que los Jefes de Estado y de Gobierno y altos representantes de diferentes países, se reúnen con un objetivo común; reafirmar las cumbres ya establecidas y pasadas en la ONU Declaración de Río sobre el Medio Ambiente y el Desarrollo, la Cumbre Mundial sobre el Desarrollo Sostenible, la Cumbre Mundial sobre Desarrollo Social, el Programa de Acción de la Conferencia Internacional sobre la Población y el Desarrollo, la Plataforma de Acción de Beijing y la Conferencia de las Naciones Unidas sobre el Desarrollo Sostenible (ONU, 2015).

Con la idea anterior se establecen las bases de una nueva era del desarrollo sostenible mediante la promulgación de 17 objetivos o metas, en adelante ODS, que alentaran durante 15 años actuaciones en cinco esferas fundamentales: las personas, el planeta, la prosperidad, la paz y las alianzas.

De esa forma se crea la nueva Agenda 2030 para el Desarrollo Dostenible fomentando la prosperidad tanto de las personas como del planeta. Se incide en dicha cumbre en que estos objetivos supondrán una continuación de los anteriores, en la que los 17 nuevos objetivos ODS reemprendan los Objetivos de Desarrollo del Milenio ODM y amplíen los mimos. En este sentido y tal como establece la Oficina Internacional del trabajo, el legado de los ODM se percibe claramente en lo ODS del 1 a 7 , por otro lado, los ODS 8 a 11 se refieren al crecimiento inclusivo y el trabajo decente, la infraestructura y la

Estudio de un caso de enseñanza de materias STEM a través del ecourbanismo apoyado por herramientas avanzadas de diseño, en el horizonte 2030 de objetivos de desarrollo sostenible (ODS). Francisco del Cerro Velázquez y Fernando Lozano Rivas. 
industrialización, la desigualdad y las ciudades, respectivamente; la temática de los ODS 12 a 15 es la protección del medio ambiente; el ODS 16 se relaciona con la gobernanza y la paz y, por último, el ODS 17 insta a revitalizar la alianza mundial en relación con las finanzas, la tecnología, la creación de capacidad, el comercio, la coherencia de las políticas, las alianzas entre múltiples interesados, y los datos, supervisión y rendición de cuentas, (OIT, 2014).

El ODS 4 "Educación de Calidad" se desglosa en 7 metas y 3 medios de implementación y ocupa, sin duda, un lugar central en la consecución de la Agenda 2030 para el Desarrollo Sostenible puesto que figura como un objetivo en sí mismo de la Agenda 2030 y porque la educación no se circunscribe al ODS 4, la educación se evoca explícitamente en las metas de los otros ODS o de alguna manera se relacionan con el resto de ODS (Del Cerro y Morales, 2018). De acuerdo con esta idea, debemos avanzar en conseguir una Educación para Todos (EPT) que a su vez sea un instrumento eficaz para alcanzar los ODS en el horizonte 2030. Por ello, en el artículo se justifica y propone el ecourbanismo como innovación metodológica en el proceso de enseñanzaaprendizaje apoyado en herramientas avanzadas de diseño que favorece la penetración y alcance social, económico y ecológico de los ODS.

\section{Objetivos de este estudio}

Se establece como objetivo general: justificar la potencialidad de la metodología innovadora propuesta (el ecourbanismo) en el proceso de enseñanza-aprendizaje de las materias STEM apoyado en herramientas de diseño avanzadas (GIMP, Goolzoom y Sketchup), a la vez que contribuye desde la educación a la penetración de los Objetivos de Desarrollo Sostenible en la sociedad.

Fijado el objetivo general los objetivos específicos del estudio podemos sintetizarlos en:

Analizar la evolución del concepto de Desarrollo Sostenible a lo largo de las distintas cumbres y conferencias internacionales hasta la actualidad en la Agenda 2030 y justificar la importancia del ODS 4 (Educación de Calidad) para alcanzar, expandir e implantar de forma efectiva el resto de ODS.

Evidenciar la potencialidad del ecourbanismo como instrumento metodológico innovador que desde la educación permitirá avanzar en el Desarrollo Sontenible, demostrando la relación o asociación entre los parámetros ecourbanos y los Objetivos de Desarrollo Sostenible.

Aplicar el instrumento metodológico planteado como una buena práctica de enseñanzaaprendizaje en asignaturas STEM, en este caso Dibujo Técnico de $1 .^{\circ}$ de bachillerato.

\section{Materiales y método}

Siguiendo los objetivos propuestos, la perspectiva del planteamiento metodológico parte, en primer lugar, de una revisión bibliográfica crítica y exahustiva sobre la evolución del concepto de desarrollo sostenible desde su primera concepción hasta la

Estudio de un caso de enseñanza de materias STEM a través del ecourbanismo apoyado por herramientas avanzadas de diseño, en el horizonte 2030 de objetivos de desarrollo sostenible (ODS). Francisco del Cerro Velázquez y Fernando Lozano Rivas. 
redacción de los 17 ODS, justificando a su vez la importancia del ODS 4 (Educación de Calidad) para alcanzar, expandir e implantar de forma efectiva el resto de ODS. En segundo lugar, se vinculan los parámetros y objetivos ecourbanos con los Objetivos de Desarrollo Sostenible, por lo que se justifica la importancia del mismo como instrumento metodológico en materias STEM. Por último, mediante el proyecto educativo "Make Cool your School", se pone en práctica la propuesta metodológica planteada.

\subsection{Desde Concepto de Desarrollo Sostenible hasta la llegada de los ODS para el horizonte 2030.}

Ya en los años 70 en Estocolmo, se siente la necesidad de un criterio y principios comunes que ofrezcan a los pueblos del mundo inspiración y guía para preservar y mejorar el medio humano, y mediante 21 principios se entiende necesario que tanto el ser humano como las instituciones tomen conciencia de la situación real del planeta y se encuentren vías alternativas para proteger la vida del ser humano en nuestro mundo (Naciones Unidas, 1976).

En el año 1977 tiene lugar la Conferencia Intergubernamental sobre Educación Ambiental organizada por la UNESCO con la colaboración del PNUMA en Tbilisi. En dicha conferencia nacen nuevas ideas de lo que es la educación ambiental y aún más importante, las dimensiones que debe tomar dicha materia. En el informe final de la Conferencia se afirma que:

La educación ambiental forma parte integrante del sistema educativo, debería girar en torno a los problemas concretos y tener un carácter interdisciplinario. Debería tender a sentar el sentido de los valores, contribuir al bienestar colectivo y preocuparse del bien estar del ser humano.

Posteriormente, en 1980, la UNESCO concreta las grandes orientaciones de la Conferencia de Tbilisi basándose en las 41 recomendaciones de la citada conferencia, estableciendo las finalidades y características pedagógicas de la educación ambiental. En este sentido, con un enfoque orientado a la resolución de problemas, la educación ambiental deberá proporcionar medios que permitan comprender las relaciones entre los diferentes factores físicos, biológicos y socioeconómicos del medio ambiente.

La característica más importante de la educación ambiental consiste probablemente en que apunta a la resolución de problemas concretos. Se trata de que los individuos, cualquiera que sea el grupo de la población al que pertenezcan y el nivel en que se sitúen, perciban claramente los problemas que coartan el bienestar individual o colectivo, diluciden sus causas y determinen los medios que pueden resolverlos. De este modo, los individuos estarán en condiciones de participar en la definición colectiva de estrategias y actividades encaminadas a zanjar los problemas que repercuten en la calidad del medio ambiente.

Por todo ello, los objetivos pedagógicos de la educación ambiental podríamos sintetizarlos en:

- Tomar conciencia del sistema natural.

- Evaluar la actividad antrópica en el medio y fomentar el análisis crítico hacia esa actividad proponiendo soluciones compatibles con la conservación del medio.

Estudio de un caso de enseñanza de materias STEM a través del ecourbanismo apoyado por herramientas avanzadas de diseño, en el horizonte 2030 de objetivos de desarrollo sostenible (ODS). Francisco del Cerro Velázquez y Fernando Lozano Rivas. 
- Propiciar un cambio actitudinal del ser humano con valores de responsabilidad encaminados a la conservación del planeta.

Es evidente que los principios u objetivos pedagógicos de la educación ambiental persiguen alcanzar un desarrollo sostenible. Sin embargo, el concepto de desarrollo sostenible o duradero, no se introduce hasta 1987, donde en el capítulo 2 "Hacia un desarrollo duradero" del Informe Brundtland, se describe este concepto como el desarrollo que satisface las necesidades de la generación presente sin comprometer la capacidad de las generaciones futuras para satisfacer sus propias necesidades (Naciones Unidas, 1987). El citado informe deja igualmente claro que el desarrollo sostenible no afecta solo al concepto medio ambiental, también implica una transformación progresiva de la economía y de la sociedad.

Esta deficinión de desarrollo sostenible se asume en la Declaración de Río sobre el Medio Ambiente y el Desarrollo, cuando su principio 3. ${ }^{\circ}$ declara "El derecho al desarrollo debe ejercerse en forma tal que responda equitativamente a las necesidades de desarrollo y ambientales de las generaciones presentes y futuras".

En septiembre de 2000, y con la base de numerosas conferencias y cumbres de las Naciones Unidas, los 189 líderes de los países miembros se reunieron en la sede en Nueva York para adoptar la Declaración del Milenio de las Naciones Unidas. Como consecuencia de esta declaración los países miembros asumieron la necesidad de conseguir en el año 2015 ocho propósitos de desarrollo humano, conocidos como los Objetivos de Desarrollo del Milenio. En el informe 2015 de los Objetivos de Desarrollo del Milenio, entre otras cuestiones, se da cuenta del compromiso alcanzado que puede resumirse en: Una reducción mundial a la mitad de personas que viven en pobreza extrema, o de cantidad de niños que en edad de recibir enseñanza primaria no asistían a la escuela, o la tasa mundial de mortalidad de niños menores de 5 años, o una reducción en un $45 \%$ de la tasa de mortalidad materna, o una reducción del $40 \%$ de nuevas infecciones del VIH entre 2000 y 2013 y, por último, compromisos relacionados con el medio ambiente tales como; recuperación de la capa de ozono, mayor acceso de agua potable a millones de personas, etc...(Naciones Unidas, 2015).

El 21 de octubre de 2015 la Asamblea General de Naciones Unidadas aprueba el documento final de la cumbre de 25 de septiembre del 2015 en el que se establece la nueva agenda para el desarrollo después de 2015 bajo el título de Transformar nuestro mundo: la Agenda 2030 para el Desarrollo Sostenible. El nuevo concepto mundial sobre Desarrollo Sostenible se enmarca dentro de los Objetivos que con ese mismo nombre "Objetivos de Desarrollo Sostenible (ODS)" se adoptan en ese documento.

En este contexto del desarrollo sostenible, el Marco de Acción Educación 2030 fue preparado por el Comité de Dirección de la EPT y aprobado en noviembre de 2015 en la 38. ${ }^{a}$ Reunión de la Conferencia General de la UNESCO. Este documento, supone la hoja de ruta específica del Objetivo de Desarrollo Sostenible 4 y, por tanto, para avanzar en el propio ODS 4 y en los demás ODS por su relación con el ODS 4.

Como resumen a este recorrido cronológico, en la figura 1 se plantea cómo el concepto inicial de desarrollo sostenible, que contiene un significado intrinseco de lo medio

Estudio de un caso de enseñanza de materias STEM a través del ecourbanismo apoyado por herramientas avanzadas de diseño, en el horizonte 2030 de objetivos de desarrollo sostenible (ODS). Francisco del Cerro Velázquez y Fernando Lozano Rivas. 
ambiental y un carácter de cambio progresivo de la economía y de la sociedad, se mantiene y se consolida decadas después con los enfoques de los ODS circunscritos en aspectos ambientales, sociales, económicos que, a su vez, interseccionan con los principios pedagógicos de la educación ambiental, de alguna manera, recogidos en los currículos de las distintas enseñanzas reguladas en las leyes educativas de los países que, en concreto, en España se recogen en la LOMCE:

... Que convierta la educación en el principal instrumento de movilidad social, ayude a superar barreras económicas y sociales y genere aspiraciones y ambiciones realizables para todos...

... El reto de una sociedad democrática es crear las condiciones para que todos los alumnos y alumnas puedan adquirir y expresar sus talentos, en definitiva, el compromiso con una educación de calidad como soporte de la igualdad y la justicia social....

... La educación tendrá por objeto el pleno desarrollo de la personalidad humana en el respeto a los principios democráticos de convivencia y a los derechos y libertades fundamentales...

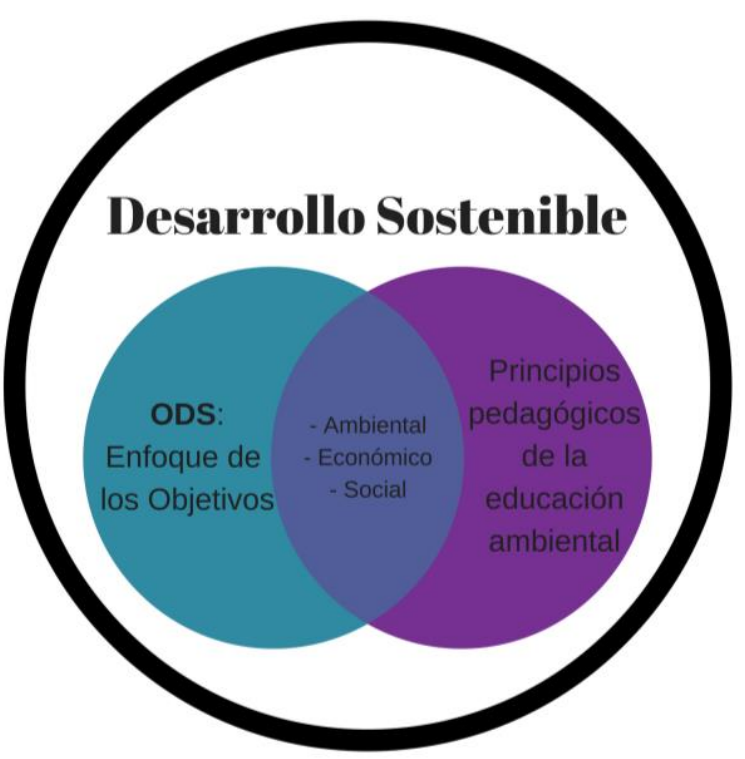

Figura 1: Intersección de los enfoques de los ODS y los principios pedagógicos de la educación ambiental.

Por tanto, es evidente que la educación es una vía fundamental para alcanzar el desarrollo sostenible. Las aulas son espacios donde los docentes pueden fomentar e inculcar valores y cambios actitudinales que permitan conseguir las metas de los ODS.

Por todo ello, es fácil entender que el ODS 4: "Educación de Calidad" es fundamental para alcanzar, expandir e implantar de forma efectiva el resto de ODS.

Por otro lado, dada la importancia del urbanismo en el desarrollo sostenible para la redacción de la Agenda 2030 fueron consultados urbanistas y, de esta forma, en la misma agenda podemos encontrar referencias como:

Reconocemos que la gestión y el desarrollo sostenibles del medio urbano son fundamentales para la calidad de vida de nuestros pueblos.

Estudio de un caso de enseñanza de materias STEM a través del ecourbanismo apoyado por herramientas avanzadas de diseño, en el horizonte 2030 de objetivos de desarrollo sostenible (ODS). Francisco del Cerro Velázquez y Fernando Lozano Rivas. 
Trabajaremos con las autoridades y las comunidades locales para renovar y planificar nuestras ciudades y asentamientos humanos con miras a fomentar la cohesión comunitaria y la seguridad de las personas y estimular la innovación y el empleo.

Reduciremos los efectos negativos de las actividades urbanas y de las sustancias químicas que son peligrosas para la salud y el medio ambiente, incluso mediante una gestión ecológicamente racional de los productos químicos y su utilización sin riesgos, la reducción y el reciclado de los desechos y un uso más eficiente del agua y la energía, y trabajaremos para minimizar el impacto de las ciudades en el sistema climático mundial.

Ya hemos visto, que sobre el ODS 4 pivota, en gran parte, el logro para alcanzar, expandir e implantar el resto de ODS y, por otro lado, también se ha justificado la importancia que la propia ONU confiere al urbanismo para contribuir en el desarrollo sostenible, por tanto, resultaría muy interesante conjugar la educación y el ecourbanismo, de tal manera que utilizando este último como instrumento metodológico de la educación, apoyado a su vez en recursos avanzados de diseño, nos permitirá poner en valor los 17 ODS.

\subsection{El Ecourbanismo como instrumento metodológico en educación: parámetros de estudio y Objetivos de Desarrollo Sostenible.}

Establecida esa relación entre desarrollo sostenible y educación, se propone que a través del ecourbanismo puedan darse soluciones a los problemas sociales, económicos y ambientales de las ciudades, por lo que éste resulta un instrumento metodológico en la educación y, fundamentalmente, en la enseñanza de materias STEM, pero también en otras como la Historia, la Geografía, etc. a la vez que se contribuye al desarrollo sostenible al educar a ciudadanos comprometidos con sus semejantes y con su entorno.

En este apartado se ofrece una nueva visión para poder, desde al aula, alcanzar los ODS utilizando el ecourbanismo como instrumento metodológico y, de esa forma, que la educación ambiental forme parte del aula.

¿Podría ser el ecourbanismo, con su estudio y puesta en práctica, un instrumento metodológico para poder enseñar y alcanzar en el aula los ODS?

Urbanistas de todas partes del mundo afirman que nos encontramos en un momento en el que los modelos de urbanismo estándar o conocidos como tradicionales no consiguen dar soluciones a los problemas sociales, económicos y ambientales de las ciudades (Chueca Goitia, 2011).

Las ineptitudes a las que nos enfrentamos no son pequeñas: el sector de la construcción acapara más de la mitad de la energía consumida en países como España; el tráfico colapsa las ciudades a diario; los espacios de relación y encuentro están desapareciendo de las ciudades, etc.

Proyectistas consideran necesario encontrar vías para un nuevo urbanismo, "Ecourbanismo", a través del impulso de la investigación y la práctica innovadora. El objetivo de estos profesionales es dar respuesta a la vez tanto a la grave situación ambiental de nuestro entorno como a las demandas de calidad de una sociedad que experimenta cambios extraordinarios día a día. Por lo tanto, hemos de dar valor las

Estudio de un caso de enseñanza de materias STEM a través del ecourbanismo apoyado por herramientas avanzadas de diseño, en el horizonte 2030 de objetivos de desarrollo sostenible (ODS). Francisco del Cerro Velázquez y Fernando Lozano Rivas. 
iniciativas que plantean, con rigor, métodos para facilitar el trabajo de los profesionales y para dar respuesta a las pretensiones de la sociedad.

A lo largo de la historia el concepto de ciudad ha ido variando centro del contexto social, económico y político del momento. No tiene nada que ver la Polis griega con la ciudad medieval; una medina musulmana es distinta de una villa cristiana, una ciudadtemplo como Pekín y una metrópoli comercial como Nueva York (Trachana, 2013).

Si nos centramos en el pensamiento de Ortega y Gasset, la ciudad por excelencia es la ciudad clásica y mediterránea, donde el elemento fundamental es la plaza. Ésta es ante todo un lugar para el diálogo, el debate y por qué no la retórica y la política. En este caso el Ágora es la sala de reunión donde las gentes parlotean, se comunican y por lo tanto se relacionan.

Hablamos de una ciudad abierta al diálogo y que, por tanto, tendrá que ver con el desarrollo de la vida ciudadana. Es evidente que para un ciudadano mediterráneo, lo principal de la ciudad sea la plaza y su significado.

Desde un punto de vista ecourbanístico, la ciudad de hoy, la gran ciudad de la sociedad industrial o postindustrial, se sitúa justo en el extremo opuesto de lo que podríamos entender por ciudad educativa.

La ciudad moderna se ve forzada a abrir vías cada vez más amplias al transporte, modificando su antigua organización, que reflejaba la acumulación de acciones de anteriores períodos históricos. Los nuevos barrios nacen ya con estos condicionantes de facilitación del tránsito y las comunicaciones. Como resultado de todo esto, la ciudad pierde diversidad de ambientes, se homogeneiza. A pesar de todo, el transporte no se resuelve, el tránsito se colapsa y el suelo del casco urbano se encarece enormemente.

La ciudad, centro de todos los intercambios, genera estrés bajo el flujo incesante, que además de saturar las calles de automóviles satura también el ambiente de humos, que los mecanismos naturales de ventilación no llegan a dispersar con eficacia suficiente. La ciudad se vuelve, además, un sistema en estrés (Girardet, 2001).

Ruidos, nerviosismo en el tránsito y prisas conducen a una patología específica. La población humana es sensible al estado de su entorno. También lo es en un sentido más mecánico, ya que la contaminación afecta a los procesos respiratorios. Todo esto son consecuencias a nivel individual del estrés del sistema.

El consumo exagerado, la excesiva concentración de gente y de intercambios en un territorio limitado son, pues, factores de estrés para el propio sistema urbano (Gaffron, 2005). Pero la conciencia de esto no va apenas más lejos que las incomodidades ligadas a los problemas del tránsito y la polución del aire a nivel local. No hay suficiente noción del papel que juegan estos focos de consumo en el conjunto del sistema mundial. En efecto, el habitante de una ciudad moderna consume una proporción de recursos materiales y de energía muy superior al consumo que tiene un habitante del Tercer Mundo. Y la desproporción se incrementa con el tiempo.

Estudio de un caso de enseñanza de materias STEM a través del ecourbanismo apoyado por herramientas avanzadas de diseño, en el horizonte 2030 de objetivos de desarrollo sostenible (ODS). Francisco del Cerro Velázquez y Fernando Lozano Rivas. 
Por otra parte, este consumo desmesurado de energía implica una aportación a la contaminación atmosférica global y, por tanto, al efecto invernadero y al cambio climático, con los peligrosos cambios que se puedan esperar, sobre todo para determinados países ya cercanos a los límites del hambre, por la alteración de las cosechas a escala mundial.

La ciudad se nos muestra así, en un nuevo aspecto, como insolidaria o incluso globalmente peligrosa (Higueras García, 2001).

También aquí vemos un punto para la reflexión educativa. La vida en la ciudad tendería a alejarnos de los comportamientos globalmente deseables, y en este sentido nos "deseducaría".

¿Qué papel juega la educación en todo esto? Hace algunos años que los docentes se han empezado a interesar por ciertos aspectos del metabolismo urbano.

Las visitas a las estaciones depuradoras de aguas residuales (EDAR), incineradoras, compañías de gas y otras instalaciones municipales y de servicios se han hecho frecuentes en los Institutos de Educación Secundaria. Se han producido materiales para el trabajo escolar sobre el agua, los desperdicios o la energía. No estamos seguros de los resultados de muchas de estas actividades, porque casi nadie evalúa seriamente los resultados pedagógicos. Se hacen las cosas y se da por bueno el resultado, casi siempre partiendo de un puro apriorismo: cualquier actividad fuera del Instituto es educación activa y seguro que se aprovecha mucho.

Para empezar, muy a menudo estas actividades están aisladas, fuera de contexto en relación con el contenido del curso, no responden a una dinámica suficientemente coherente que persiga la sensibilización y la formación, la mejor comprensión de estos aspectos ambientales del fenómeno urbano. La comprensión de la problemática ambiental y del significado de la ciudad en este contexto exige un planteamiento global que ha de impregnar el proceso educativo.

No es posible limitarse a unas clases más, como las que se aplican para enseñar una técnica, por ejemplo estudiar circuitos eléctricos en la materia de Tecnología. Aquí se trata de una educación cívica y crítica, de la transmisión de unos nuevos valores morales además de unos conocimientos concretos.

Pero si esto es ya una limitación bastante seria, hay que añadir que muchas actividades fuera del Instituto no están suficientemente pensadas. Falta a menudo motivaciones por parte de los alumnos, que no saben con certeza qué van a ver, ni tienen un trabajo concreto que realizar durante la visita. Y, lo que es todavía más grave, a menudo es el mismo profesor quien carece de motivación y abandona la tarea en manos de los monitores que conducen la visita.

Pero no es ahora el momento de hacer un análisis de limitaciones y dificultades que se encuentran en todas las actividades de educación ambiental. Lo que conviene destacar es que, precisamente porque nos enfrentamos con un problema de educación cívica, la

Estudio de un caso de enseñanza de materias STEM a través del ecourbanismo apoyado por herramientas avanzadas de diseño, en el horizonte 2030 de objetivos de desarrollo sostenible (ODS). Francisco del Cerro Velázquez y Fernando Lozano Rivas. 
responsabilidad no puede recaer solamente en el Instituto. La sociedad y la ciudad han de actuar de marco educativo.

Han de educar para cambiar. Aunque lo parezca, esto no es un círculo vicioso, sino un proceso de aproximaciones sucesivas. De hecho, hemos visto nacer una oferta educativa no escolar, en forma de equipamientos: museos, centros del medio urbano o de la naturaleza, vías verdes, etc. Es una oferta que nace del tejido social y se dirige en primer lugar a la escuela, pero también a otros públicos. Los medios de comunicación, los órganos de la administración, las asociaciones de consumidores o entidades privadas muy variadas invaden así, con una oferta, el mundo de la educación. Según el contenido de los mensajes que generen, actuarán de una manera regresiva, acentuando los problemas que hemos ido mencionando, o, al contrario, ayudarán a superarlos. En un sentido u otro, la ciudad es educadora.

¿Influirá el ecourbanismo en la educación urbana de los alumnos en las aulas?

La metodología que se presenta en es la siguiente:

En primer lugar, se exponen cuales son los parámetros ecourbanísticos que se han de trabajar para poder realizar un proyecto ecourbano. En segundo lugar, se relacionarán con los objetivos ecourbanísticos que se persiguen alcanzar y, finalmente, se relacionan éstos y los ODS.

La planificación y, por lo tanto, el desarrollo de una Ecociudad abarca cuatro componentes básicos (Gaffron, 2008).

\section{- Configuración urbana:}

Es la realidad física de la ciudad, considerada como un sistema interconectado. Las subcategorías asociadas a este sector de cara al proceso de planificación son: La demanda de suelo, el uso del suelo, las zonas verdes, el bienestar urbano, el espacio público y la edificación.

\section{- Materiales y energía:}

Referida al movimiento de energía y materia en el espacio y a través de los diferentes sistemas físicos y urbanos. Las subcategorías asociadas a este sector de cara al proceso de planificación son: La energía, el agua, los residuos y los materiales de construcción.

\section{- Transportes:}

Relacionada con el movimiento físico de personas, mercancías e información dentro y fuera de la ciudad. Las subcategorías asociadas a este sector de cara al proceso de planificación son: Medios de transporte no motorizados, transporte público, transporte motorizado individual y el transporte de mercancías.

\section{- Aspectos sociales y económicos:}

Vinculados a las actividades humanas que determinan los procesos sociales y la vida económica de la ciudad.

Estudio de un caso de enseñanza de materias STEM a través del ecourbanismo apoyado por herramientas avanzadas de diseño, en el horizonte 2030 de objetivos de desarrollo sostenible (ODS). Francisco del Cerro Velázquez y Fernando Lozano Rivas. 
En la tabla 1 se muestran los parámetros ecourbanísticos en la creación de un proyecto urbano sostenible.

Tabla 1. Parametros ecourbanísticos en la creación de un proyecto urbano sostenible

Parámetros ecourbanísticos en la creación de un proyecto urbano sostenible.

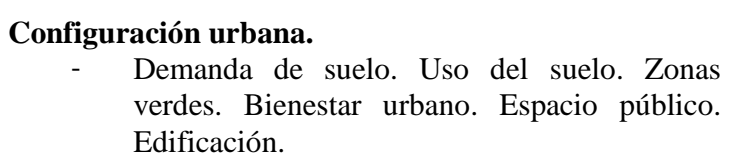

Demanda de suelo. Uso del suelo. Zonas verdes. Bienestar urbano. Espacio público. Edificación.

\section{Transportes.}

- Transporte público. Transporte de mercancías.

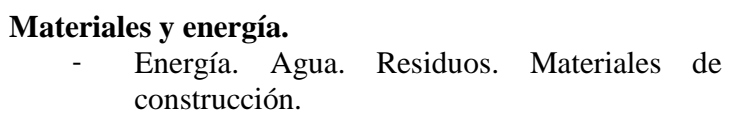

- Energía. Agua. Residuos. Materiales de construcción.

Aspectos Sociales.

- $\quad$ El hombre es un ser de relación. Es necesaria una planificación urbana que fomente dichas relaciones

\section{Fuente: Ecocity Book I. A better place to live (2005)}

Nos preguntamos: ¿Cómo podemos trabajar los objetivos ecourbanísticos? lo haremos a través de los parámetros antes citados.

Como se puede observar en la tabla 2, los objetivos ecourbanísticos agrupados en tres grandes grupos (aspectos ecológicos, aspectos económicos y aspectos sociales) se relacionan con los parametros ecourbanísticos en la creación de un proyecto urbano sostenible (Lozano Rivas, 2011).

Tabla 2. Relación entre los objetivos ecourbanisticos y los parámetros ecourbanísticos en la creación de un proyecto urbano sostenible.

Objetivos ecourbanísticos

\section{Aspectos ecológicos}

- $\quad$ Ciudad de recorrido compendioso.

- Elección del área de actuación.

- Medios de transporte ambientales.

- Descenso de la contaminación atmosférica.

- $\quad$ Descenso del nivel de ruido.

- Aprovechamiento de la luz natural.

- Fomento de la actividad física: carril bici ...

Reutilización de las aguas residuales.
Parámetros de estudio

\section{Configuración urbana.}

- Demanda de suelo.

- Uso del suelo.

- Zonas verdes.

- Bienestar urbano.

- $\quad$ Espacio público.

- Edificación.

\section{Materiales y energía.}

- Energía.

- $\quad$ Agua.

$\overline{\text { Estudio de un caso de enseñanza de materias STEM a través del ecourbanismo apoyado por herramientas }}$ avanzadas de diseño, en el horizonte 2030 de objetivos de desarrollo sostenible (ODS). Francisco del Cerro Velázquez y Fernando Lozano Rivas. 


\begin{tabular}{|c|c|c|c|}
\hline & Aspectos económicos & - & Residuos. \\
\hline- & Materiales ecológicos. & & Materiales de construcción \\
\hline- & Energías verdes. & & Transportes. \\
\hline- & Peatonalización. (conservación de vías). & - & Medios de transporte no motorizados. \\
\hline- & Beneficios ofrecidos por la calidad ambiental. & - & Transporte público.. \\
\hline- & Ahorro energético & - & Transporte de mercancías. \\
\hline & Aspectos sociales & & Aspectos Sociales. \\
\hline- & $\begin{array}{l}\text { Accesibilidad y supresión de barreras } \\
\text { arquitectónicas. } \\
\text { Ciudad de densidad moderada: Se favorece la } \\
\text { diversidad social e interacción humana. }\end{array}$ & - & $\begin{array}{l}\text { El hombre es un ser de relación. Es necesaria } \\
\text { una planificación urbana que fomente dichas } \\
\text { relaciones } \\
\text { Aspectos económicos. }\end{array}$ \\
\hline- & $\begin{array}{l}\text { Zonas verdes. Interacción social. } \\
\text { Movilidad. }\end{array}$ & - & $\begin{array}{lccc}\text { Es } \quad \text { necesario } \quad \text { conectar } & \text { con } & \text { los } \\
\text { intereses reales de la población } & & \end{array}$ \\
\hline
\end{tabular}

Como hemos enunciado con anterioridad son 17 los ODS que las Naciones Unidas promulgaron en septiembre de 2015 para el horizonte 2030. ¿Existe algún vínculo entre las propuestas ecourbanísticas y estos objetivos?

Estos objetivos entraron en vigor el 01 de enero de 2016 y son muchos los desafíos a los que se enfrenta cada país; desigualdades sociales, naturales, económicas y, por tanto, es necesaria una reconciliación con el planeta respetando los enfoques de cada país. En este sentido, es interesante señalar que la ONU establece o indica que cada país, podrá utilizar los instrumentos que estime oportunos para poder llevar a cabo la hazaña. En este sentido, se reconoce que cada país dispone de diferentes enfoques, visiones de futuro, modelos e instrumentos para lograr el desarrollo sostenible, en función de sus circunstancias y prioridades nacionales, y reafirmamos que el planeta Tierra y sus ecosistemas son nuestro hogar común y que "Madre Tierra" es una expresión corriente en muchos países y regiones.

Como ya se ha justificado a lo largo de la investigación existe una relación directa entre los objetivos del ecourbanismo y los ODS, en tanto que en ambos casos los aspectos de fondo son ecológicos, económicos y sociales. Con esta idea, en la tabla 3 se muestra esa relación entre objetivos ecourbanísticos y ODS.

Tabla 3. Relación entre los objetivos ecourbanos y los ODS

$$
\text { Objetivos ecourbanos Objetivos de desarrollo sostenible }
$$

\begin{tabular}{lll}
\hline & \multicolumn{1}{c}{ Aspectos ecológicos } & \\
- & Ciudad de recorrido compendioso. & ODS 3 Salud y Bienestar. \\
- & Elección del área de actuación. & ODS 6 Agua limpia y saneamiento. \\
- & Medios de transporte ambientales. & ODS 7 Energía asequible y no contaminante. \\
- & Descenso de la contaminación atmosférica. & ODS 11 Ciudades y comunidades sostenibles. \\
- & Aprovechamiento de ruido. & Oa luz natural. \\
- & Fomento de la actividad física: carril bici ... & ODS 12 Producción y consumo responsable. \\
\hline
\end{tabular}

Estudio de un caso de enseñanza de materias STEM a través del ecourbanismo apoyado por herramientas avanzadas de diseño, en el horizonte 2030 de objetivos de desarrollo sostenible (ODS). Francisco del Cerro Velázquez y Fernando Lozano Rivas. 
- $\quad$ Reutilización de las aguas residuales.

ODS 13 Acción por el clima.

ODS 14 Vida submarina

ODS 15 Vida de ecosistemas terrestres.

ODS 17 Alianzas para conseguir los objetivos.

\section{Aspectos económicos}

Materiales ecológicos.

Energías verdes.

Peatonalización. (conservación de vías).

Beneficios ofrecidos por la calidad ambiental.

Ahorro energético.
ODS 8 Trabajo decente y crecimiento económico.

ODS 9 Industria innovación e infraestructura.

ODS 2 Hambre cero.

ODS 17 Alianzas para conseguir los objetivos.

\section{Aspectos sociales}

- Accesibilidad y supresión de barreras arquitectónicas.

- Ciudad de densidad moderada: Se favorece la diversidad social e interacción humana.

- Zonas verdes. Interacción social.

- Movilidad
ODS 1 Fin de la pobreza.

ODS 4 Educación de calidad.

ODS 5 Igualdad de género.

ODS 10 Reducción de las desigualdades.

ODS 16 Paz, justicia e instituciones solidarias.

ODS 17 Alianzas para conseguir los objetivos.

El instrumento está perfectamente entrelazado y en el siguiente apartado se presenta el boceto de un proyeto desarrollado por alumnos de $1 .^{\circ}$ de Bachillerato, utilizando el ecourbanismo como recurso metodológico apoyado en recursos TIC emergentes.

\subsection{Caso del Proyecto educativo: Espacios de oportunidad ecourbanos en tu centro.}

El proyecto desarrollado surge como consecuencia de la convocatoria de la I Olimpiada de Arquitectura de la Región de Murcia (2018) "Make Cool Your School”. El objetivo es ofrecer a los alumnos, una experiencia en aprendizaje a través de proyectos y trabajo cooperativo, de manera que partiendo de las instalaciones de su centro, participen en el proyecto y diseño de nuevos espacios sostenibles, en los que se pueda aprender mejor juntos. En concreto, participaron en el proyecto educativo, tal y como establecía la normativa del concurso, alumnos pertenecientes al colegio La Inmaculada de Cartagena de $1 .^{\circ}$ de bachillerato en la materia de Dibujo Técnico.

Los espacios de convivencia son clave para mejorar la experiencia del aprendizaje, facilitar las interacciones, ser flexibles ante la variedad de necesidades, impulsar la creatividad, y, por supuesto, para fomentar el uso de las TIC. La percepción espacial que ofrecen las TIC, como la Realidad Aumentada, ayudan a razonar en numeros campos y a la hora de realizar esquemas, gráficos o croquis (Del Cerro y Morales, 2017). En esta experiencia, los alumnos son los responsables del rediseño de estos espacios de oportunidad, incorporándose su visión en el futuro del centro tal y como se expone en las bases del citado concurso.

Estudio de un caso de enseñanza de materias STEM a través del ecourbanismo apoyado por herramientas avanzadas de diseño, en el horizonte 2030 de objetivos de desarrollo sostenible (ODS). Francisco del Cerro Velázquez y Fernando Lozano Rivas. 
Bajo el acicate que supone para los alumnos participar en un concurso, trabajaron con una metodología constructivista y, tras un análisis de la situación real de su centro de enseñanza, proyectaron nuevos espacios sostenibles teniendo en cuenta los tres aspectos señalados por la ONU relacionados con los ODS: sociales, económicos y ecológicos. Para ello, aplicaron las habilidades y técnicas adquiridas en las materias de Dibujo Técnico y Tecnología, así como, la potencialidad que les ofrecen las tecnologías emergentes en el campo de la cartografía y el CAD. Este tipo de herramientas TIC han sido determinantes para poder llevar a cabo el proyecto educativo.

Es evidente que las TIC han transformado la configuración de espacios y de entornos físicos y tecnológicos Zapata-Ros (2014), así como nuestra experiencia urbana y, por tanto, este nuevo paradigma interfiere en el ámbito del diseño urbano.

La vida urbana cada vez es más tecnológica, esto ya es una realidad. Las tecnologías digitales conviven con nosotros, abriendo una nueva capa superpuesta en el espacio físico: "lo digital". En esta era digital y IV Revolución Industrial, las ciudades son el escenario dónde todo interacciona y se concentran experiencias, se comparten herramientas tecnológicas, laboratorios urbanos, actividades, se promueven iniciativas de co-creación- participación, y se intercambian datos, información, en definitiva, conocimiento y creatividad (Sanz Palau 2016).

A continuación, se va a describir brevemente las fases generales mediante las cuales se ha proyectado el nuevo espacio de oportunidad en el centro educativo.

En las figuras 2 y 3 se observa a los alumnos con el boceto y la concepción de la idea sobre la transformación del espacio. La primera fase del proyecto permite a los alumnos trabajar de manera cooperativa mediante un brainstorming, valorando soluciones posibles, nuevos puntos de vista y, de esta manera, potenciar el efecto multiplicador (combinación de ideaciones y sus mejoras).

El uso de las TIC está presente en todas las fases del proyecto. En este caso, los alumnos han utilizado el GeoPortal Urbanismo Cartagena; una aplicación web mediante la cual se ha trabajado toda la información cartográfica de la zona de actuación, planos, ortofotos, así como imágenes satélite. Han imprimido dichos planos y sobre el papel han ido realizando anotaciones sobre las ideas planteadas.

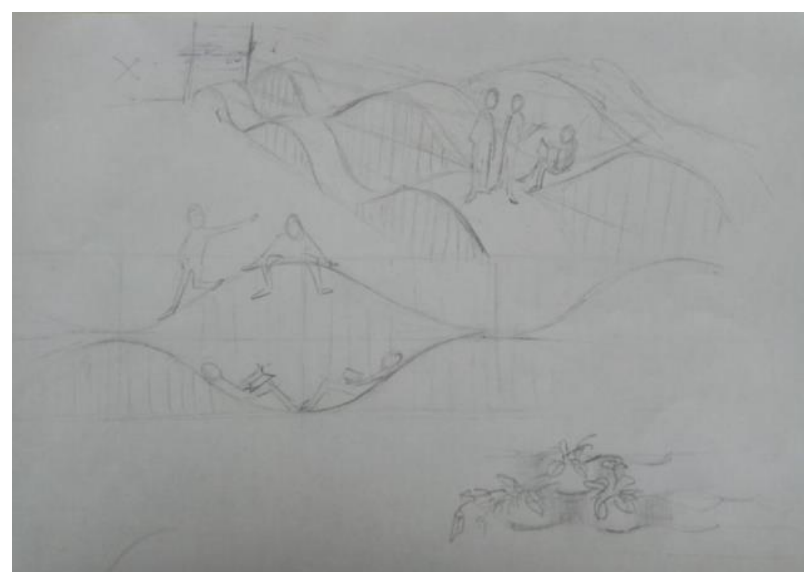

Figura 2: Concepción de la idea sobre los futuros espacios de oportunidad.

Estudio de un caso de enseñanza de materias STEM a través del ecourbanismo apoyado por herramientas avanzadas de diseño, en el horizonte 2030 de objetivos de desarrollo sostenible (ODS). Francisco del Cerro Velázquez y Fernando Lozano Rivas. 


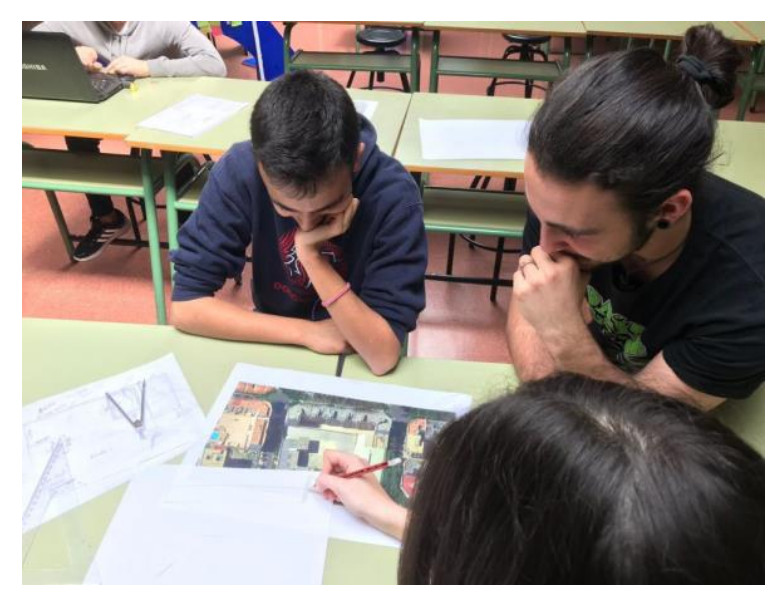

Figura 3: Alumnos trabajando sobre el boceto y las ortofotos.

En las figuras 4 y 5 se observa a los alumnos en la segunda fase del proyecto utilizando el program GIMP de edición de imágenes digitales en forma de mapas de bits, de esta manera retocan las fotografías que previamente se habían tomado de los espacios del centro. Todo proyecto urbanístico consta de un anejo fotográfico. Los alumnos mediante las TIC mejoran la calidad y aspecto deseado de las mismas. Dichas imágenes retocadas formarán parte del anejo, y también permitirán enriquecer tanto los planos como la memoria descriptiva del proyecto.

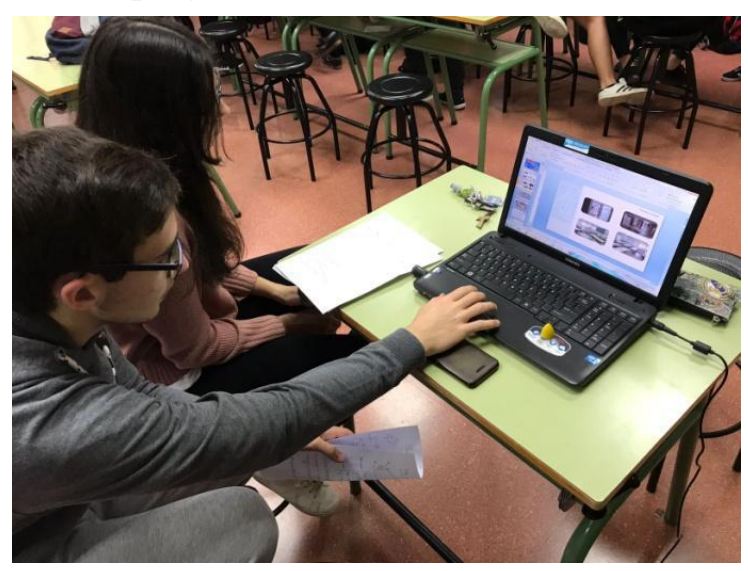

Figura 4: Alumnos retocando las fotografías con el programa GIMP.

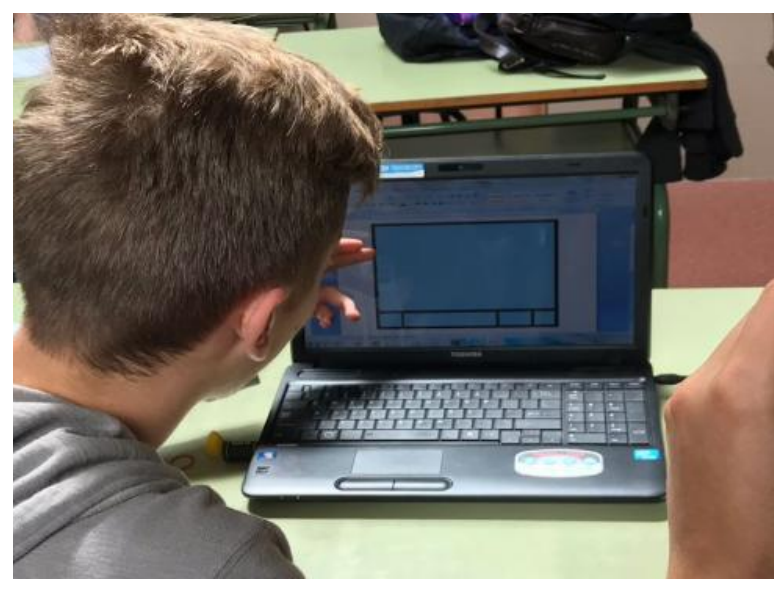

Figura 5: Alumnos montando las fotografías con el programa GIMP.

Estudio de un caso de enseñanza de materias STEM a través del ecourbanismo apoyado por herramientas avanzadas de diseño, en el horizonte 2030 de objetivos de desarrollo sostenible (ODS). Francisco del Cerro Velázquez y Fernando Lozano Rivas. 
En las figuras 6 y 7 los alumnos proyectan digitalmente el diseño del nuevo espacio de oportunidad mediante dos herramientas avanzadas de diseño: Goolzoom Mapas con el que se ha trabajado la cartografía en planta, cotas, escalas y perfiles longitudinales del terreno así como la elaboración de los planos de situación, emplazamiento y planta de estado actual y, por otro lado, utilizando Google Sketchup como programa de diseño gráfico y modelado en tres dimensiones mediante el cual se ha realizado un diseño tridimensional del espacio de oportunidad y, por tanto, en el que han desarrollado su competencia digital.

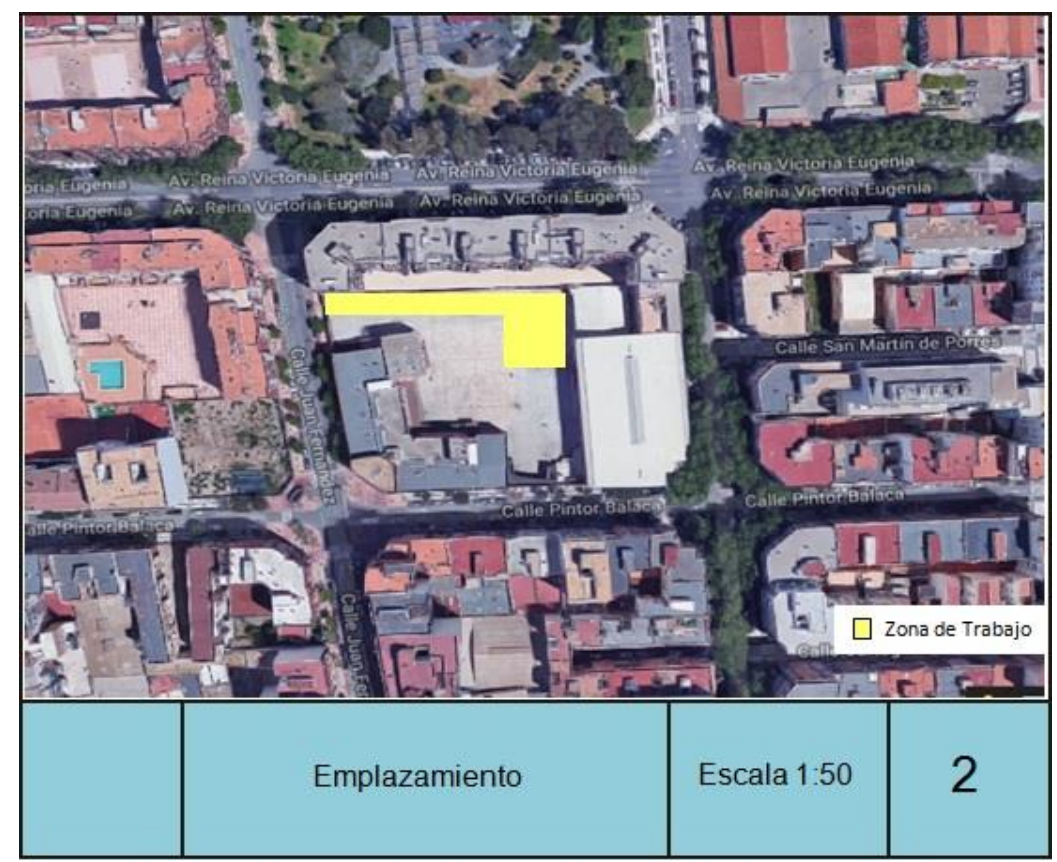

Figura 6: Estudio del terreno mediante Goolzoom.

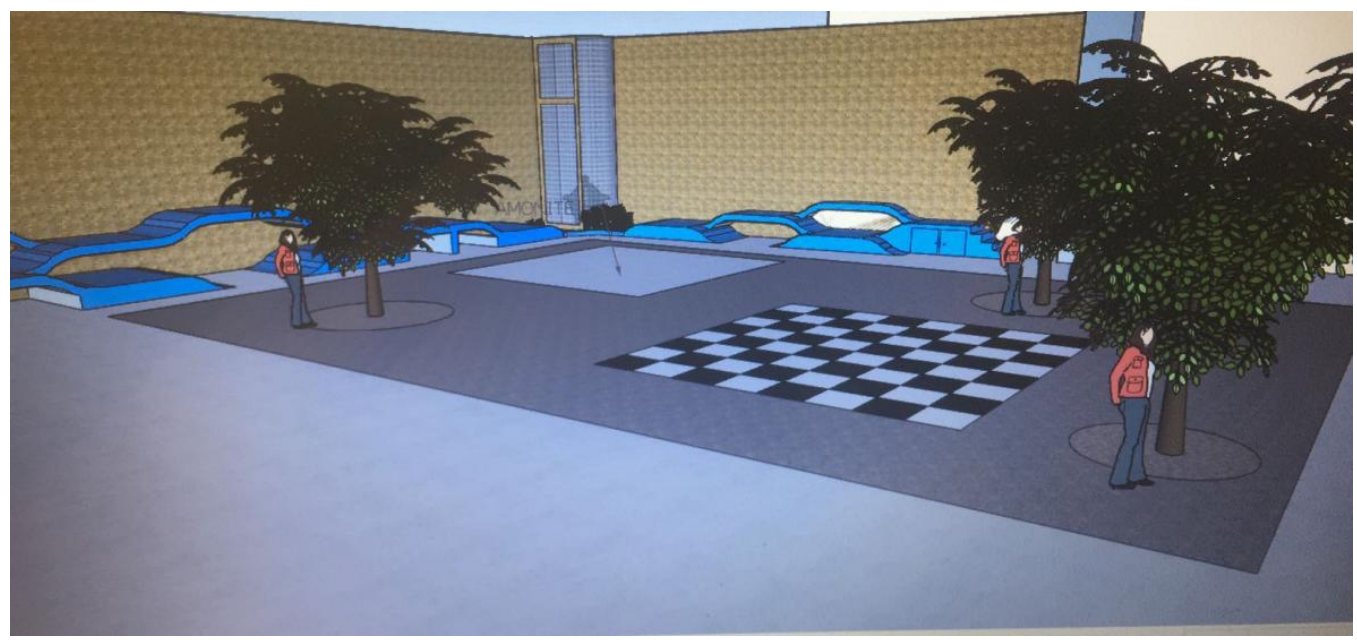

Figura 7: Diseño en planta del espacio proyectado mediante Sketchup.

\section{Resultados}

En el artículo se ha justificado la importancia del ODS 4 (Educación de Calidad) para alcanzar, expandir e implantar de forma efectiva el resto de ODS y se ha evidenciado la

Estudio de un caso de enseñanza de materias STEM a través del ecourbanismo apoyado por herramientas avanzadas de diseño, en el horizonte 2030 de objetivos de desarrollo sostenible (ODS). Francisco del Cerro Velázquez y Fernando Lozano Rivas. 
potencialidad del ecourbanismo como instrumento metodológico que desde la educación permitirá avanzar en el desarrollo sostenible mediante un aprendizaje basado en proyectos $(\mathrm{ABP})$, en este caso proyectos ecourbanos.

Se ha planteado cómo el concepto inicial de desarrollo sostenible se mantiene y se consolida decadas después con los enfoques de los ODS circunscritos en aspectos ambientales, sociales, económicos que, a su vez, interseccionan con los principios pedagógicos de la educación ambiental. Dada esta evidente intersección o relación entre desarrollo sostenible y educación, ya indicada en el párrafo anterior, se ha evidenciado y propuesto el ecourbanismo, desde la educación, para dar soluciones a los problemas sociales, económicos y ambientales de las ciudades.

Finalmente, como aplicación del ecourbanismo como instrumento metodológico apoyado en recursos TIC emergentes, se presenta el ejemplo de un proyecto dirigido a alumnos de educación secundaria en el que se trabajan los ODS y que facilita mediante una metodología constructivista, el aprendizaje y la evaluación de los alumnos en materias STEM (Science, technology, Engineering and mathematics). Mediante este instrumento metodológico, alumnos de Dibujo Técnico de $1 .^{\circ}$ de Bachillerato han afianzado contenidos de los 3 bloques establecidos en el Decreto Curricular (Decreto 221/2015, de 2 de septiembre): Geometría y Dibujo Técnico, Sistemas de representación y Normalización. De esta forma, el alumno ha podido movilizar e interrelacionar contenidos vinculados directamente con los bocetos, croquis y planos.

El proyecto ha permitido evaluar numerosos estándares de aprendizaje de $1 .^{\circ}$ de bachillerato, estando previsto un estudio empírico para cuantificar los estándares de aprendizaje en el área de Dibujo Técnico, consideramos que el ecourbanismo permitiría además la evaluación de estándares en las distintas áreas de conocimiento STEM.

El proyecto desarrollado fue fallado con el primer premio de la I Olimpiada de Arquitectura de la Región de Murcia (2018). "Make Cool Your School".

\section{Discusión}

Sobre el ODS 4 pivota, en gran parte, el logro para alcanzar, expandir e implantar el resto de ODS y, por otro lado, la propia ONU confiere al urbanismo una importancia relevante para contribuir en el desarrollo sostenible, por ello, al conjugar la educación y el ecourbanismo apoyado en recursos TIC, ponemos en valor los 17 ODS y contribuímos desarrollo de las nuevas generaciones, con una actitud crítica y positiva con el desarrollo sostenible.

El ecourbanismo llevado al aula nos ha permitido desarrollar una metodología de trabajo cuyo resultado ha sido, entre otros, un aumento en las capacidades del alumno. Por otro lado, el carácter instrumental del Dibujo Técnico ha permitido a los alumnos trabajar la interdisciplinariedad, la creatividad, la iniciativa, el trabajo en equipo y el pensamiento crítico, aptitudes puestas en valor en el currículo (Decreto 221/2015, de 2 de septiembre). Sin embargo, no solo debemos esperar a que las nuevas generaciones se desarrollen con una actitud crítica y positiva con el desarrollo sostenible y que en un futuro ocupen puestos de responsabilidad, también es necesario un compromiso por parte de los países integrantes para con la nueva agenda.

Estudio de un caso de enseñanza de materias STEM a través del ecourbanismo apoyado por herramientas avanzadas de diseño, en el horizonte 2030 de objetivos de desarrollo sostenible (ODS). Francisco del Cerro Velázquez y Fernando Lozano Rivas. 
Desde una perspectiva social, son los gobiernos de los distintos países los que deben poner en valor prácticas eficaces que fomenten una visión perdurable y real de las ciudades y del desarrollo sostenible.

Hoy en día las empresas son parte fundamental en el crecimiento económico de un país y su responsabilidad para con el desarrollo sostenible es vital. Su actitud debe ser ser crítica y responsable, implicándose de manera íntegral en los asuntos sociales, económicos y ambientales que nos rodean.

\section{Conclusiones}

El recorrido cronológico sobre las distintas Cumbres, Conferencias y/o Declaraciones de la ONU sobre Medio Humano, Medio Ambiente, Educación Ambiental, Desarrollo, etc. nos ha permitido constatar y concluir como el concepto inicial de Desarrollo Sostenible del Informe Brundtland 1987 que engloba tres factores: ambiental, económico y social, se ha mantenido y consolidado hasta la actualidad de forma que los enfoques de los ODS aprobados por la ONU en 2015 se circunscriben en aspectos ambientales, sociales, económicos, como también lo hacen los principios pedagógicos de la educación ambiental, por lo que una "Educación de Calidad" (ODS 4) es vía fundamental para alcanzar el Desarrollo Sostenible.

En este sentido, ha resultado muy interesante conjugar la educación y el ecourbanismo, de tal manera que utilizando este último como instrumento metodológico de la educación, apoyado a su vez en recursos TIC, nos ha permitido poner en valor los 17 ODS. Con esta idea o propuesta del ecourbanismo como instrumento metodológico en la educación, para contribuir al Desarrollo Sostenible al educar a ciudadanos comprometidos con sus semejantes y con su entorno, concluimos que existe una relación directa entre los objetivos ecourbanisticos y los parámetros ecourbanísticos en la creación de un proyecto urbano sostenible y, así, se han relacionado, a la vez, que también se han relacionado los objetivos ecourbanísticos y los ODS.

Desde la observación del caso planteado, tenemos el convencimiento de que este tipo de proyectos permite fomentar en los alumnos, principios de aprendizaje como: la disposición y el efecto motivador al trabajar con herramientas con las que están habituados o por el propio hecho de la competición, la intensidad en el aprendizaje por el realismo con el mundo que les rodea al proyectar y diseñar un nuevo espacio de oportunidad para su centro educativo y saber mantener un diálogo entre la teoría y la experimentación, el trabajo colaborativo asumiendo responsabilidades y el pensamiento crítico.

Como ya se ha citado, son cada vez más los autores que opinan que la ciudad actual se nos muestra insolidaria o incluso globalmente peligrosa. De acuerdo con esto, la vida en la ciudad tendería a alejarnos de los comportamientos globalmente deseables, y en este sentido nos "deseducaría".

Otros autores ya han considerado propuestas ecourbanas como viables:

The "Smart City" concept revolves around the idea of embodying cutting-edge information and communication technology solutions in the very fabric of future cities

Estudio de un caso de enseñanza de materias STEM a través del ecourbanismo apoyado por herramientas avanzadas de diseño, en el horizonte 2030 de objetivos de desarrollo sostenible (ODS). Francisco del Cerro Velázquez y Fernando Lozano Rivas. 
to offer new and better services to citizens while lowering the city management costs in monetary, social, and environmental terms (ISWCS, 2018)

Estocolmo es una de las capitales pioneras en la proyección de ecourbanismo. Pasear por sus calles es admirar in situ, entornos de oportunidad, donde nuevos y ecológicos materiales muestran trazados innovadores que se materializan en plazas, parques, avenidas y viviendas eco. Un claro ejemplo es la simbiocity, un enfoque holístico del desarrollo urbano sostenible en la que el gobierno sueco a través de su Oficia de Comercio toma esta iniciativa con el objetivo de ahondar en el desarrollo sostenible a nivel mundial. Funciones urbanas, energía sostenible, gestión de residuos, suministro de agua y servicios sanitarios, tráfico y transporte, planificación del paisaje y el diseño de edificios son algunos de los ejemplos que sustentan las ideas que desarrollamos en el presente artículo (Simbiocity, 2018).

Si entendemos el urbanismo como un elemento que nos permite diseñar, proyectar y construir barrios y ciudades solamente desde un punto de vista técnico, entonces vivimos tiempos difíciles.

Vivimos en un mundo complejo, donde la sociedad humana continúa siendo percibida como algo alejado que no nos compromete directamente, pero no debe ser así.

Las ciudades pueden adoptar nuevos e imaginativos enfoques en la planificación y gestión del transporte y en el uso del suelo urbano, en definitiva, crear nuevos barrios más habitables, reduciendo el deseo de las personas de querer escapar de la presión de la vida de la ciudad. Necesitamos alcanzar una concepción de las ciudades en las que estas sean lugares de creatividad y convivencia para la vida íntima en comunidad y de esta manera, las ciudades puedan educar a los ciudadanos.

Si hacemos las cosas bien, las ciudades del nuevo milenio serán centros para una cultura sostenible. Serán eficientes en la gestión de la energía y de los recursos, agradables para las personas, culturalmente ricas y dotadas de democracias activas que aseguren que se hace el mejor uso de las facultades humanas.

Pero nada de esto ocurrirá si no creamos un equilibrio entre lo material y lo espiritual. Es imprescindible tener una visión más reposada y serena de las ciudades para ayudarlas a consumar su pleno potencial como lugares no solo del cuerpo sino también del espíritu.

Fecha de presentación del artículo: 1 de septiembre de 2018

Fecha de aprobación: 11 de septiembre de 2018

Fecha de publicación: 30 de septiembre de 2018

Del Cerro Velázquez, F. y Lozano Rivas, F. (2018). Estudio de un caso de enseñanza de materias STEM a través del ecourbanismo apoyado por herramientas avanzadas de diseño, en el horizonte 2030 de objetivos de desarrollo sostenible (ODS). RED. Revista de Educación a Distancia, 58. Consultado el (dd/mm/aaaa) en http://www.um.es/ead/red/

Estudio de un caso de enseñanza de materias STEM a través del ecourbanismo apoyado por herramientas avanzadas de diseño, en el horizonte 2030 de objetivos de desarrollo sostenible (ODS). Francisco del Cerro Velázquez y Fernando Lozano Rivas. 


\section{Financiación}

La experiencia presentada en esta investigación ha recibido el fallo del tribunal como primer premio, dotado de una financiación de $400 €$, de la I Olimpiada de Arquitectura de la Región de Murcia 2018 "Make Cool Your School”. Olimpiada convocada por la Universidad Politécnica de Cartagena y patrocinada por: Comunidad Autónoma de la Región de Murcia, Fundación Séneca, Olimpiadas Cientifica de la Región de Murcia, Universidad Politécnica de Cartagena y la Escuela Técnica Superior de Arquitectura y Edificación de Cartagena.

\section{Referencias.-}

Del Cerro, F. y Morales, G. (2017). Realidad aumentada como herramienta de mejora de la inteligencia especial en estudiantes de educación secundaria. Revista de Educación a Distancia, Núm.54.

Del Cerro, F. y Morales, G. (2018). Augmented Reality and Mobile Devices: A Binominal Methodological Resource for Inclusive Education (SDG 4). An example in Secondary Education. Sustainability 2018, 10 (10), 3446;

https://doi.org/10.3390/su10103446

Decreto: 221/2015, por el que se establece el currículo del Bachillerato en la Comunidad Autónoma de la Región de Murcia. Boletín Oficial de la Región de Murcia, núm. 203, 03 de septiembre de 2015, pp. 31594-32545.

Chueca Goitia, F. (2011). Breve historia del urbanismo. Madrid: Alianza Editorial.

Gaffron, P. (2005). Ecocity Book I. A better place to live. Viena: Facultas Verlags- and Buchahandels

Gaffron, P. (2008). Ecocity Book II. How to make it happen. Viena: Facultas Verlagsand Buchahandels

GeoPortal Urbanismo Cartagena: Ayuntamiento de Cartagena. Área de Urbanismo. Recuperado de: http://geo.cartagena.es/gemuc_004/

Gimp. GNU Image Manipulation Program. (2.10.6). Recuperado de: http://www.gimp.org.es/

Girardet, H. (2001). Creando ciudades sostenibles. Valencia: Tilde.

Goolzoom Mapas. Recuperado de: https://es.goolzoom.com/Mapas.aspx7

Higueras García, E. (2001). Urbanismo y medio ambiente: La ciudad. El microclima urbano y el bienestar. Madrid: Cuadernos del Instituto Juan de Herrera de la Escuela de Arquitectura de Madrid.

Estudio de un caso de enseñanza de materias STEM a través del ecourbanismo apoyado por herramientas avanzadas de diseño, en el horizonte 2030 de objetivos de desarrollo sostenible (ODS). Francisco del Cerro Velázquez y Fernando Lozano Rivas. 
International Symposium on Wireless Communication Systems. Recuperado de: http://iswcs2018.org/

La ciudad sostenible. Manual de diseño. Madrid: IDAE.

Ley Orgánica 8/2013, de 9 de diciembre, para la mejora de la calidad educativa. Boletín Oficial del Estado, 295, de 10/12/2013, pp. 97858-97859. Recuperado de: https://www.boe.es/buscar/act.php?id=BOE-A-2013-12886

Lozano Rivas, F. (2011). Ecourbanismo, innovación urbana y social en la ciudad. Material no publicado.

Naciones Unidas. (1972, 5-16 de junio). Declaración de la Conferencia de las Naciones Unidas sobre el Medio Humano 5-16 de junio de 1972 (Informe de la conferencia de las Naciones Unidas sobre el Medio Humano. 1973). Recuperado de:

https://www.dipublico.org/conferencias/mediohumano/A-CONF.48-14-REV.1.pdf

Naciones Unidas. (1987). Informe Brundtland de la Comisión Mundial sobre Medio Ambiente y Desarrollo. Recuperado de:

http://www.exteriores.gob.es/Portal/es/PoliticaExteriorCooperacion/Desarrollososte nible/Documents/Informe\%20Brundtland\%20(En\%20ing1\%C3\%A9s).pdf

Naciones Unidas. (1992, 3-14 de junio). Declaración de Rió sobre el Medio Ambiente y el Desarrollo, del 3 al 14 de junio de 1992. Recuperado de:

http://www.un.org/spanish/esa/sustdev/documents/declaracionrio.htm

Naciones Unidas. (2000, 13 de septiembre). Declaración del milenio. Recuperado de: http://www.un.org/spanish/milenio/ares552.pdf.

Naciones Unidas. (2015). Informe de los Objetivos de Desarrollo del Milenio de 2015. Disponible en:

http://www.un.org/es/millenniumgoals/pdf/2015/mdg-report-2015_spanish.pdf

Naciones Unidas. (2015, 25 de septiembre). Transformar nuestro mundo: la Agenda 2030 para el Desarrollo Sostenible. Recuperado de:

https://unctad.org/meetings/es/SessionalDocuments/ares70d1_es.pdf

OIT. (2014, 19 de septiembre). Acta 322 de la Oficina Internacional del Trabajo. GB.322/INS/6 "La agenda para el desarrollo sostenible después de 2015: Actualización" 14 de septiembre de 2014. Recuperado de: http://www.ilo.org/wcmsp5/groups/public/---ed_norm/--relconf/documents/meetingdocument/wcms_311035.pdf

ONU. (2015, 25 de septiembre). Resolución 70/01 de la Asamblea General Transformar nuestro mundo: La agenda 2030 para el Desarrollo Sostenible A/RES/70/. Recuperado de: http://www.un.org/es/comun/docs/?symbol=A/RES/70/1

Estudio de un caso de enseñanza de materias STEM a través del ecourbanismo apoyado por herramientas avanzadas de diseño, en el horizonte 2030 de objetivos de desarrollo sostenible (ODS). Francisco del Cerro Velázquez y Fernando Lozano Rivas. 
Sanz Palau, H. (2016, 04 de marzo). Sobre como las TIC han transformado nuestra experiencia urbana. Recuperado de: http://www.urbanlivinglab.net/tic-urbana/

Simbiocity (s.f). Sustainability by Sweden. Recuperado de: http://www.simbiocity.se

Sketchup. Recuperado de: https://www.sketchup.com/es

Trachana, A. (2013). La ciudad histórica: Una genealogía del espacio publico. Madrid: Cuadernos del Instituto Juan de Herrera de la Escuela de Arquitectura de Madrid.

UNESCO. (1978, abril). Informe final de la Conferencia intergubernamental sobre educacion ambiental. Recuperado de:

http://unesdoc.unesco.org/images/0003/000327/032763sb.pdf

UNESCO. (1980). Las grandes orientaciones de la Conferencia de Tbilisi. Recuperado de: http://unesdoc.unesco.org/images/0003/000385/038550so.pdf

Zapata-Ros, M. (2014) La configuración de espacios y de entornos físicos y tecnológicos en la nueva Enseñanza Superior (I). Redes abiertas http://redesabiertas.blogspot.com.es/2014/04/la-configuracion-de-espacios-yde.html 